\title{
Use of a New Algorithm with an Internally Cooled Electrode for Radiofrequency Ablation of Small Hepatocellular Carcinomas*
}

\author{
Ho V. Nguyen ${ }^{1 \#}$, Kathleen A. Khong ${ }^{1}$, John P. McGahan ${ }^{1}$, Kai Ding ${ }^{2}$, Machelle D. Wilson ${ }^{2}$ \\ ${ }^{1}$ Department of Radiology, University of California Davis Medical Center, Sacramento, USA \\ ${ }^{2}$ Department of Public Health Sciences, University of California, Davis, USA \\ Email: "1eavesam@gmail.com
}

Received November 8, 2013; revised December 8, 2013; accepted December 15, 2013

Copyright (C) 2013 Ho V. Nguyen et al. This is an open access article distributed under the Creative Commons Attribution License, which permits unrestricted use, distribution, and reproduction in any medium, provided the original work is properly cited. In accordance of the Creative Commons Attribution License all Copyrights (C) 2013 are reserved for SCIRP and the owner of the intellectual property Ho V. Nguyen et al. All Copyright (C) 2013 are guarded by law and by SCIRP as a guardian.

\begin{abstract}
Purpose: To investigate the efficacy of a new algorithm to increase the volume of tissue ablation via gradual ramp-up of power using an internally cooled electrode for ablating hepatomas $3 \mathrm{~cm}$ or less. Materials and Methods: 44 patients with 62 hepatomas were treated from March 4, 2004 to May 24, 2009. Ablation with a gradual ramp-up of power was performed using a single needle with an internally cooled electrode. Evaluation for tumor response was performed with 4-phase CT at 24 hours and 3 months. All immediate and follow-up complications were recorded. Results: Complete tumor ablation was achieved in $86 \%$. The ablation volumes were $16 \mathrm{~cm}^{3}+/-12 \mathrm{~cm}^{3}$ for tumors $<2 \mathrm{~cm}$ and $28 \mathrm{~cm}^{3}+/-$ $12 \mathrm{~cm}^{3}$ for tumors $2-3 \mathrm{~cm}$. Out of 68 total ablation sessions, there were 2 major complications (pleural effusions) requiring intervention (thoracentesis). Conclusion: Compared with existing techniques using a constant full-power setting, ablation of small hepatomas using an algorithm of gradual ramp-up of power provides comparable rate of complete tumor ablation, adequate ablation volumes, and a low rate of complications that require treatment.
\end{abstract}

Keywords: Hepatocellular Carcinoma; Hepatoma; Radiofrequency Ablation; Internally-Cooled Electrode; Ramp-up; Full-Power; Algorithm

\section{Introduction}

Hepatocellular carcinoma (HCC) is one of the most common and lethal cancers in the world with rising incidence in Western countries. Most cases of HCC arise from pre-existing cirrhosis. Currently, liver transplant and surgical resection offer the best chances for survival. Those who are not transplant or surgical candidates may undergo percutaneous radiofrequency ablation (RFA), using ultrasound (US) or computed tomography (CT) guidance.

Radiofrequency ablation of HCC has undergone many refinements in coagulation algorithm and needle design. One design uses an internally cooled electrode (ICE) with an uninsulated tip, which was observed by Goldberg

\footnotetext{
${ }^{*}$ The statistical analysis portion of this project was supported by the National Center for Advancing Translational Sciences (NCATS), National Institutes of Health (NIH), through grant \#UL1 TR000002.

${ }^{\#}$ Corresponding author.
}

et al. to allow for "increased power deposition without tissue charring" [1].

Early experiments with the "cooled-tip" electrode advocate applying maximum current with pulsed-energy delivery for 12 minutes or until the generator reaches full impedance [1-7]. The manufacturer of the "cooled-tip" electrode and RF generator has recommended this as the standard protocol, one which has been utilized in subsequent experiments. For expandable, non-saline-perfused electrodes, there are treatment algorithms that start at a low power setting, which gradually increases [8]. However, this approach has not been done with internally cooled electrodes. A recent ex vivo experiment on bovine livers was performed with a "cooled-tip" electrode to determine technical parameters that maximize tissue coagulation. The experiment shows that one can increase tissue ablation by gradually ramping up power and repeating ablations after reaching maximum power im- 
pedance [9].

In this retrospective study, we investigate the in vivo efficacy of this algorithm using a single "cooled-tip" electrode for lesions with average diameter $\leq 3 \mathrm{~cm}$.

\section{Materials and Methods}

\subsection{Patient Characteristics}

From March 4, 2004 to May 24, 2009, 44 consecutive patients with $62 \mathrm{HCCs}$, each of which measuring $\leq 3 \mathrm{~cm}$ in average diameter, underwent RFA with a single "cooled-tip" electrode at the radiology department of the University of California Davis Medical Center. Patient characteristics are summarized in Table 1. Among these, 27 were males and 17 were females. The mean age was 60 years $+/-11$ years. 37 patients had hepatitis C, 2 had hepatitis B, 3 had alcoholic cirrhosis, and 2 had cirrhosis of unknown etiology. 32 patients had single tumors, 6 had 2 tumors each, and 6 had 3 tumors each. Average tumor diameter ranged $0.9 \mathrm{~cm}$ to $3.0 \mathrm{~cm}$, with a mean of $2.0 \mathrm{~cm}+/-0.6 \mathrm{~cm}) .62$ total tumors were ablated. We then compare our results (ablation volume, rate of com-

Table 1. Baseline patient and tumor characteristics.

\begin{tabular}{|c|c|}
\hline Characteristic & Value \\
\hline Number of patients & 44 \\
\hline Age (years) & $60+/-11$ \\
\hline \multicolumn{2}{|l|}{ Gender } \\
\hline Male & 27 \\
\hline Female & 17 \\
\hline \multicolumn{2}{|l|}{ Liver Disease } \\
\hline Hepatitis C & 37 \\
\hline Hepatitis B & 2 \\
\hline Alcoholic cirrhosis & 3 \\
\hline Cirrhosis, unknown cause & 2 \\
\hline \multicolumn{2}{|l|}{ MELD* Category } \\
\hline 1 & 25 \\
\hline 2 & 17 \\
\hline 3 & 2 \\
\hline \multicolumn{2}{|l|}{ Number of Tumors } \\
\hline 1 & 32 \\
\hline 2 & 6 \\
\hline 3 & 6 \\
\hline Tumor Size (cm) & $2.0+/-0.6$ \\
\hline
\end{tabular}

*MELD-Model for End-Stage Liver Disease. plete tumor ablation, complications) to other published studies that used a full-power algorithm.

Patients were included following institutional review board approval. Informed consent was waived in this retrospective analysis. Exclusion criteria were: (1) presence of tumors $>3 \mathrm{~cm}$ in average diameter, (2) extrahepatic disease, (3) tumor invasion into the main or lobar portal vein. Diagnosis of HCC, as shown on CT or Magnetic Resonance Imaging (MRI), was based on arterial hypervascularity in a lesion $>1 \mathrm{~cm}$ with venous or delayed phase washout in accordance with American Association for the Study of Liver Diseases (AASLD), which takes the view that a lesion found incidentally or on screening in a patient with known hepatitis B or cirrhosis of other etiology is likely to be hepatocellular carcinoma [10].

\subsection{Pre-Procedural Imaging}

Analysis of preoperative imaging and patient selection were performed by the physician (JPM) performing the RFA in conjunction with referring physicians at weekly hepatic tumor board conferences. An ultrasound examination and either CT or MRI were performed in all patients for pre-operative planning. This included a fourphase liver CT/MR with a base scan, an arterial phase (delay of approximately 25 - 30 seconds), portal phase (delay of approximately $70-80$ seconds), and equilibrium phase (delay of approximately 180 seconds). Outside CT/MRI, stored in a separate server in DICOMM format, within 6 weeks of the planned procedure were acceptable if they were of adequate technical quality, as determined by the physician performing the RFA.

The lengths, widths, and heights of the masses were measured from the CTs or MRIs using the axial, sagittal, and coronal images and from the ultrasound using the transverse and longitudinal images. The volumes were calculated by using the formula for volume of an ellipsoid: $0.52 \times$ length $(\mathrm{L}) \times$ width $(\mathrm{W}) \times$ height $(\mathrm{H})$.

\subsection{Treatment Methods}

Informed consent was obtained on all patients before the procedures. All ablations were performed by a single physician (JPM) with over 10 years of hepatic RFA experience to limit physician variables that may contribute to the rates of technical success or complications. An RF generator ( $\mathrm{CC} 1$; Covidien, Boulder, Colo) capable of producing $200 \mathrm{~W}$ and a 17 -gauge needle (Covidien) with a 3-cm exposed tip were used in every ablation.

All procedures were performed under general anesthesia. Each tumor was localized with ultrasound immediately prior to the initiation of RFA. Needle placement was performed under ultrasound guidance using the Acuson Sequoia unit (Mountain View, CA) with a 4 
$\mathrm{MHz}$ sector transducer. For lesions difficult to visualize under ultrasound, CT was used for guidance or verification of needle placement, most commonly a 4-MDCT Toshiba Aquilion scanner (Tustin, CA). CT fluoroscopy was never used, at the operator's preference, to minimize radiation exposure to the operator, patient, and personnel.

Each ablation started with the power setting at $50 \mathrm{~W}$. The power was gradually increased by $10 \mathrm{~W}$ each minute using the impedance control mode. RFA was not stopped at 12 minutes or when the generator turned off after reaching maximum power impedance ("roll-off'). Rather, the generator was restarted and ablation continued until 3 total "roll-offs" occurred [9]. Needle position was readjusted between ablations for lesions $2-3 \mathrm{~cm}$ but not for lesions $\leq 2 \mathrm{~cm}$.

At the end of each treatment, color Doppler ultrasound was performed to detect any "color line" sign following electrode removal, which would indicate bleeding [11]. Patients were admitted overnight. Any adverse events were evaluated and recorded. A 4-phase liver CT was performed the morning following the procedure. If there were no adverse events, patients were discharged the day after treatment.

\subsection{Post-Treatment Assessment and Follow-up}

The zone of ablation, defined by the nonenhancing region on the portal phase, was evaluated at the 24-hour $\mathrm{CT}$. The length (L), width (W), and height $(\mathrm{H})$ were measured for each zone of ablation. The volume was calculated using the formula for volume of an ellipsoid $(0.52 \times \mathrm{L} \times \mathrm{W} \times \mathrm{H})$.

Evaluation for tumor response was performed after analysis of 4-phase CT at 24 hours and 3 months. Complete response is defined as satisfying the following criteria on both studies: (1) the zone of ablation extends beyond the borders of the original tumor; (2) absence of nodular enhancement within or at the periphery of all treated tumors on both arterial and portal phases and absence of washout on the delayed phase.

All immediate and follow-up complications were recorded. We reviewed the procedure note, 24-hour CT scan, admission note, discharge summary, and outpatient follow-up clinic visits. Minor and major complications were classified according to Society of Interventional Radiology guidelines. A complication was classified as minor if it did not require therapy or an extended hospital stay. A major complication is one that required transfusion, interventional radiology or surgical management, or extended hospital stay for observation [12].

\section{Results}

\subsection{Technical Success}

During the initial RFA session, 62 tumors underwent ablation. On the 24-hour study, only 3 tumors demonstrated evidence of residual tumor. However, on the 3 month post-ablation scan, evidence of residual tumor was identified in 6 other lesions, giving a total of 9 lesions out of 62 that demonstrated evidence of residual at 3 months. This gives a complete ablation rate of 53/62 $(86 \%)$.

For tumors with average diameter $\leq 2 \mathrm{~cm}$ (with average volume of $2 \mathrm{~cm}^{3}$ ), the ablation volume was $16 \mathrm{~cm}^{3}+/-$ $12 \mathrm{~cm}^{3}$. For tumors with average diameter $2 \mathrm{~cm}$ to $3 \mathrm{~cm}$ (average volume $8 \mathrm{~cm}^{3}$ ), the ablation volume was $28 \mathrm{~cm}^{3}$ $+/-12 \mathrm{~cm}^{3}$. The ablation volume was at least 3 times greater than the tumor volume in 52/62 (84\%) tumors, and at least 2 times greater in 57/62 (92\%) tumors.

\subsection{Complications}

A total of 68 RFA sessions were performed, with 28 patients receiving 1 RFA session, 9 patients receiving 2 RFA sessions, 6 patients receiving 3 RFA sessions, and 1 patient receiving 4 RFA sessions. Residual tumors and local or distant tumor progression often underwent repeat ablations. Major complications occurred in 2 sessions. They were both symptomatic right pleural effusion that required therapeutic thoracentesis. An additional 14 sessions had minor complications (small pleural effusions, subcapsular fluid, minor increase in ascites, or self-limited fever secondary to tumor lysis) that did not require therapeutic intervention or extended hospital stay.

\section{Discussion}

The goal of radiofrequency ablation is to create adequate treatment margins to reduce the risk of local tumor progression. RFA technique has been refined over the years to include different types of electrodes and algorithms to increase volume of ablation. In clinical practice, increasing the ablation volume may result in fewer electrode placements, thereby decreasing procedural time and risks associated with needle repositioning. Various needle designs have been used for ablation of HCC including expandable electrodes, needles with side-holes to allow saline perfusion into the tumor, ICEs, and internally cooled wet (ICW) electrodes (saline is used for both cooling the electrode internally and for perfusion into the tumor) $[4,5,13,14]$. Initial experiments demonstrated increased coagulation volumes with ICEs compared with nonperfused electrodes $[1,15]$. The ICW electrodes, not investigated in our study, has been shown to produce larger ablation volumes in ex vivo bovine and in vivo porcine livers compared with ICEs [14].

With the ICE, ablation volume is further increased by using pulsed energy delivery [16]. For the "cooled-tip" electrode RF system, the standard protocol has been the method recommended by the manufacturer of applying 
maximum power with pulsed-energy delivery for 12 minutes or until the generator reached full impedance. However, one can maximize volume of coagulation necrosis by continuing ablation beyond this point. Applying a clustered electrode to in vivo muscle, Goldberg et al. showed that by increasing the RF duration from 12 to 30 minutes, the coagulation diameter increased from $7.2 \mathrm{~cm}$ to $9.5 \mathrm{~cm}$ [16]. A similar increase was also seen for ex vivo liver. As coagulation volume is a cubic function of diameter, a $2.3 \mathrm{~cm}$ increase in coagulation diameter more than doubles the coagulation volume [16]. More recently, ex vivo research in the bovine liver model has shown that by gradually ramping up power and repeating ablations after reaching maximum power impedance for more than 12 minutes, the total volume of ablation was increased. In fact, by use of gradual ramp up of power and restarting ablations after maximum impedance, ablation volume increased by as much as eight times [9]. However, this was an ex vivo experiment. To our knowledge, there are no experiments validating these results in clinical practice.

In this experiment, we found that ablation volumes obtained using a "cooled-tip" electrode with a gradual ramp-up of power provided adequate zones of ablation for tumors $<3 \mathrm{~cm}$. In $84 \%$ of cases, we obtained an ablation volume at least 3 times greater than that of tumor volume. The studies we reviewed that utilized ICEs for ablating small lesions did not report ablation volumes; therefore, a direct comparison to our results cannot be made. However, our ablation volumes $\left(16 \mathrm{~cm}^{3}+/-12\right.$ $\mathrm{cm}^{3}$ for tumors $<2 \mathrm{~cm}$, and $28 \mathrm{~cm}^{3}+/-12 \mathrm{~cm}^{3}$ for tumors $2-3 \mathrm{~cm}$ ) were comparable to those obtained from ICW electrodes for both tumors $<2 \mathrm{~cm}\left(14.5 \mathrm{~cm}^{3}+/-5.1\right.$ $\left.\mathrm{cm}^{3}\right)$ and tumors $2-3 \mathrm{~cm}\left(29.3 \mathrm{~cm}^{3}+/-10.9 \mathrm{~cm}^{3}\right)$ [13]. ICW electrodes have been shown to have higher ablation volumes compared with ICEs in a recent experiment using ex vivo bovine and in vivo porcine liver models [14]. Consequently, our results may indirectly suggest that the gradual ramp-up of power algorithm increases volume of ablation obtained with the ICE. However, this should be confirmed by a direct comparison of ablation volumes obtained with an ICE using the two algorithms. This may be of clinical significance as a rim of coagulation of normal liver is necessary to ensure complete ablation [17]. If ending the RFA after 12 minutes results in a small zone of ablation, multiple ablations, requiring repeated electrode placements, may be needed to completely ablate the tumor [18].

The rate of complete tumor ablation at the initial RFA session $(86 \%)$ is comparable to existing studies which define technical success following one RFA session. A prospective study, using an expandable electrode and full-power algorithm had a rate of $83 \%$ complete ablation at 1 month with tumor size of $2.8 \mathrm{~cm}+/-0.7$ [3]. A ret- rospective study using expandable electrode and fullpower technique had $88.6 \%$ rate of complete ablation in tumors $<3 \mathrm{~cm}$, determined by 2 observations $>4$ weeks apart [2]. Two studies, using ICEs and full-power technique, had technical success rates of 95\% [5] and 96\% [4]. However, one study defined technical success following up to 2 RFA sessions [4], and the other up to 3 RFA sessions [5].

Our study also shows that RFA using a single "cooledtip" electrode with a gradual ramp up of power is safe with few major complications. The two major complications that occurred in 68 RFA sessions were both large right pleural effusions which resolved with therapeutic thoracentesis. In one case, the hospitalization was increased by 2 days; however, patient also had infectious pneumonitis. In the second case, the patient was discharged the day after RFA and admitted 2 days later for management of a hemothorax, which was treated with therapeutic thoracentesis. The 14 minor complications were small pleural effusions, subcapsular fluid, minor increase in ascites, or self-limited fever secondary to tumor lysis, that did not require treatment or additional days of hospitalization. These findings are consistent with the low rate of complications that has been found on previous studies using the full-power algorithm [2-5,13].

Limitations of the current study include: inclusion of patients treated at only a single medical center, the lack of a control group, the small number of patients, and the retrospective nature of the study.

\section{Conclusion}

Compared with the full-power technique, radiofrequency ablation of small hepatomas using a new algorithm of gradual ramp-up of power provides a comparable rate of complete ablation, adequate ablation volumes, and a low rate of major complications.

\section{REFERENCES}

[1] S. N. Goldberg, G. S. Gazelle, L. Solbiati, W. J. Rittman and P. R. Mueller, "Radiofrequency Tissue Ablation: Increased Lesion Diameter with a Perfusion Electrode," Academic Radiology, Vol. 3, No. 8, 1996, pp. 636-644. http://dx.doi.org/10.1016/S1076-6332(96)80188-7

[2] A. Guglielmi, A. Ruzzenente, M. Sandri, et al., "Radio Frequency Ablation for Hepatocellular Carcinoma in Cirrhotic Patients: Prognostic Factors for Survival," Journal of Gastrointestinal Surgery: Official Journal of the Society for Surgery of the Alimentary Tract, Vol. 11, No. 2, 2007, pp. 143-149.

http://dx.doi.org/10.1007/s11605-006-0082-y

[3] R. Lencioni, D. Cioni, L. Crocetti, et al., "Early-Stage Hepatocellular Carcinoma in Patients with Cirrhosis: Long-term Results of Percutaneous Image-Guided Radiofrequency Ablation," Radiology, Vol. 234, No. 3, 2005, 
pp. 961-967.

[4] O. Seror, G. N'Kontchou, M. Tin-Tin-Htar, et al., "Radiofrequency Ablation with Internally Cooled versus Perfused Electrodes for the Treatment of Small Hepatocellular Carcinoma in Patients with Cirrhosis," Journal of Vascular and Interventional Radiology: JVIR, Vol. 19, No. 5, 2008, pp. 718-724. http://dx.doi.org/10.1016/j.jvir.2008.01.007

[5] T. Shibata, T. Shibata, Y. Maetani, H. Isoda and M. Hiraoka, "Radiofrequency Ablation of Small Hepatocellular Carcinoma: A Prospective Comparison of Internally Cooled Electrode and Expandable Electrode," Radiology, Vol. 238, No. 1, 2006, pp. 346-353.

http://dx.doi.org/10.1148/radiol.2381041848

[6] S. Shiina, R. Tateishi, T. Arano, et al., "Radiofrequency Ablation for Hepatocellular Carcinoma: 10-Year Outcome and Prognostic Factors," The American Journal of Gastroenterology, Vol. 107, No. 4, 2011, pp. 569-577. http://dx.doi.org/10.1038/ajg.2011.425

[7] K. Yan, M. H. Chen, W. Yang, et al., "Radiofrequency Ablation of Hepatocellular Carcinoma: Long-Term Outcome and Prognostic Factors," European Journal of Radiology, Vol. 67, No. 2, 2008, pp. 336-347. http://dx.doi.org/10.1016/j.ejrad.2007.07.007

[8] S. M. Lin, C.J. Lin, H. J. Chung, C. W. Hsu and C. Y. Peng, "Power Roll-Off During Interactive Radiofrequency Ablation Can Enhance Necrosis When Treating Hepatocellular Carcinoma," American Journal of Roentgenology, Vol. 180, No. 1, 2003, pp. 151-157. http://dx.doi.org/10.2214/ajr.180.1.1800151

[9] J. P. McGahan, S. Loh, F. J. Boschini, et al., "Maximizing Parameters for Tissue Ablation by Using an Internally Cooled Electrode," Radiology, Vol. 256, No. 2, 2010, pp. 397-405. http://dx.doi.org/10.1148/radiol.09090662

[10] J. Bruix and M. Sherman, "Management of Hepatocellular Carcinoma: An Update," Hepatology, Vol. 53, No. 3, 2011, pp. 1020-1022. http://dx.doi.org/10.1002/hep.24199

[11] J. P. McGahan, L. Wright and J. Brock, "Occurrence and Value of the Color Doppler 'Line Sign' after Radiofrequency Ablation of Solid Abdominal Organs," Journal of Ultrasound in Medicine: Official Journal of the American
Institute of Ultrasound in Medicine, Vol. 30, No. 11, 2011, pp. 1491-1497.

[12] D. Sacks, T. E. McClenny, J. F. Cardella and C. A. Lewis, "Society of Interventional Radiology Clinical Practice Guidelines," Journal of Vascular and Interventional Radiology Vol. 14, No. 9, 2003, pp. S199-S202. http://dx.doi.org/10.1097/01.RVI.0000094584.83406.3e

[13] J. H. Kim, P. N. Kim, H. J. Won and Y. M. Shin, "Percutaneous Radiofrequency Ablation Using Internally Cooled Wet Electrodes for the Treatment of Hepatocellular Carcinoma," AJR American Journal of Roentgenology, Vol. 198, No. 2, 2012, pp. 471-476. http://dx.doi.org/10.2214/AJR.11.6583

[14] J. Cha, D. Choi, M. W. Lee, et al., "Radiofrequency Ablation Zones in Ex Vivo Bovine and In Vivo Porcine Livers: Comparison of The Use of Internally Cooled Electrodes and Internally Cooled Wet Electrodes," Cardiovascular and Interventional Radiology, Vol. 32, No. 6, 2009, pp. 1235-1240. http://dx.doi.org/10.1007/s00270-009-9600-0

[15] S. N. Goldberg, P. F. Hahn, K. K. Tanabe, et al., "Percutaneous Radiofrequency Tissue Ablation: Does Perfusion-Mediated Tissue Cooling Limit Coagulation Necrosis?" Journal of vascular and Interventional Radiology: JVIR, Vol. 9, No. 1, 1998, pp. 101-111. http://dx.doi.org/10.1016/S1051-0443(98)70491-9

[16] S. N. Goldberg, L. Solbiati, P. F. Hahn, et al., "LargeVolume Tissue Ablation with Radio Frequency by Using a Clustered, Internally Cooled Electrode Technique: Laboratory and Clinical Experience in Liver Metastases," Radiology, Vol. 209, No. 2, 1998, pp. 371-379.

[17] J. P. McGahan and G. D. Dodd, "Radiofrequency Ablation of the Liver: Current Status," AJR American Journal of Roentgenology, Vol. 176, No. 1, 2001, pp. 3-16. http://dx.doi.org/10.2214/ajr.176.1.1760003

[18] G. D. Dodd, M. S. Frank, M. Aribandi, S. Chopra and K. N. Chintapalli, "Radiofrequency Thermal Ablation: Computer Analysis of the Size of the Thermal Injury Created by Overlapping Ablations," AJR American Journal of Roentgenology, Vol. 177, No. 4, 2001, pp. 777-782. http://dx.doi.org/10.2214/ajr.177.4.1770777 